\title{
Impact of shading on a flat CPV system for façade integration
}

Solar Energy 140 (2016) 162-170

\author{
L.A.A. Bunthof ${ }^{a}$, F.P.M. Kreuwel ${ }^{b}$, A. Kaldenhoven ${ }^{a}$, S. Kin ${ }^{b}$, W.H.M. Corbeek $^{a}$, G.J. Bauhuis ${ }^{a}$, \\ E. Vlieg ${ }^{a}$, and J.J. Schermer ${ }^{a}$ \\ ${ }^{a}$ Radboud University, Institute for Molecules and Materials, Applied Materials Science, Heyen- \\ daalseweg 135, 6525 AJ, Nijmegen, The Netherlands \\ ${ }^{b}$ Wellsun, Molengraafsingel 12, 2629 JD, Delft, The Netherlands
}

\begin{abstract}
Recently adopted energy efficiency policies in the EU induce a movement towards energy-neutral buildings. Building integrated photovoltaics technology connects with this ambition, as aside from the generation of electrical energy, it allows additional benefits such as heat generation, or daylight regulation by transmission of diffuse sunlight through transparent parts of the system. In this study three Concentrator Photocoltaic (CPV) system configurations that allow for the construction of semi-transparent building façade elements are investigated outdoor. The systems are a Fresnel lens based concentrator, a novel flat planar optic concentrator, and a 4x4 panel of these flat optics. The flat optic has no air cavity to account for optical focal depth which is highly beneficial when incorporated in a window. In particular the energy production of the systems when partially shaded is investigated, as adjoining systems will move behind one another during sun tracking, because the optics spacing must be small to achieve good daylight regulation. The planar optic concentrator is found to display similar performance as a Fresnel lens based concentrator of similar concentration. For a multi-receiver panel, shading introduces a loss of performance ranging from 7 to $12 \%$ which is attributed to electrical interconnection as individual receivers do not suffer this loss.
\end{abstract}

\section{Keywords}

Concentrator photovoltaics, Building integration, Inhomogeneous illumination, Local spectral variation

\section{Introduction}

Forty per cent of the European energy consumption is attributed to buildings [1]. The European Union actively adopts energy efficiency policies to reduce this amount and has defined in the Directive on the Energy Performance of Buildings the "20-20-20" objectives: $20 \%$ decrease in greenhouse gas emissions, $20 \%$ share of renewable energy and $20 \%$ improvement in energy efficiency by 2020. Building-Integrated Photovoltaic (BIPV) systems address these goals perfectly. Among BIPV technologies, Building-Integrated Concentrating Photovoltaic (BICPV) systems possess additional features that make them particularly interesting for building integration, such as the possibility of heat generation [2], or daylight regulation [3]. Also the replacement of expensive semi-conductor cell area by cheaper and more environmentally friendly concentrating optics can make for a more viable system in terms of both cost and environment [4]. On the other hand it should be noted that the light-to-electricity efficiency is generally smaller for BICPV systems because of optical losses, uncollected diffuse irradiation etc. However, in the particular case of window integrated CPV, this uncollected insolation can be transmitted inside, providing 
natural lighting while converting the blinding direct component into electricity. In recent years, there has been an increase in BICPV for integration in either the roof or the façade. Chen et. al. designed a diffusive solar cell window which transfers solar radiation to solar cells at the edge of the window [5]. Aste et. al. show a new generation luminescent solar concentrator (LSC) for façade integration. They show a better energy performance ratio for the LSC compared with standard PV modules [6]. Gomes et. al. studied shading in asymmetric Photovoltaic Thermal (PVT) collectors caused by oblique solar angles and found that impact by shading can be reduced by transparent end gables, as well as by reducing the cell area [2]. Sharma and Mallick discuss a dielectric compound parabolic concentrator suited for building integration in higher latitude locations. Real time outdoor performance of the concentrator is compared to a non concentrating flat plate and the superior output power of the concentrator is shown both for sunny and rainy days [7]. Zacharopoulos et. al. have shown that at higher sun tilt angles, non-imaging dielectric linear concentrators for façade integration collects far more solar radiation than a flat plate PV of the same area [8]. Baig et.al. discuss several low concentration systems for building integration. Emphasis is placed on non-uniformity in illumination and temperature across the PV as well as detailed modeling and performance analysis of the systems [9-11]. Chemisana et. al. designed a holographic concentrator for building integration that protects the solar cell from overheating as the infrared is not concentrated in this setup. They find that the use of the concentrator increases the efficiency of the PV cell by 3\%. Also they designed a façade integrated PVT collector based on two reflecting strips and stationary PV. They show the increased performance of this system compared to reference $[12,13]$. Voarino et. al. introduce a CPVT system for roof integration that relies on a prism combined with a parabilic mirror that rotate separately to track the sun instead of using a heavy and bulky tracking system [14]. Many of these systems are designed to be stationary with low concentration, while others are designed for high concentration. Higher concentration systems have a low acceptance angle, thus are more restrictive towards the use of the diffuse part of the insolation and often require sun-tracking to function properly, yet they also offer the greatest reduction in cell area which allows for good cost-efficiency. Additionally, because the bright direct fraction of light is concentrated to the $\mathrm{PV}$ while diffusive light is not concentrated in high concentration setups (as detailed in figure 1), these offer the best opportunity to include a daylight regulation functionality, which will be the focus of the systems discussed in this work.

In this paper we study the concept of incorporating CPV into a building façade to act primarily as a window with an incorporated daylight regulation functionality, as well as a source of energy. This concept entails the removal of the harsh direct illumination by the system to be converted into energy, while allowing natural lighting of the building interior, as diffuse light passes through transparant parts of the system. Three model CPV systems are considered in this context. Firstly a common Fresnel based system with a triple junction (TJ) cell having a geometric concentration factor of 100. Secondly, a novel planar optic based high concentration receiver which relies on internal reflections to guide direct sunlight to an integrated TJ cell [15]. The prime benefit of this receiver for BICPV is that it is flat and does not feature an air cavity to account for optical focal depth, as opposed to a Fresnel lens based system. The receiver has a high geometrical concentration factor of 920; we will show that in practice, the actual concentration factor typically exceeds 600. Therefore this system has the potential for much higher electrical output than existing BIPV systems that typically do not exceed concentration factors of 50, whilst also providing daylight regulation functionality. Finally a $4 \times 4$ panel of these receivers is considered. As for all high concetration PV applications, precise tracking of the sun is required $[16,17]$, as the optic of these systems is designed to transfer direct illumination to a small high-efficiency TJ III-V solar cell. Such cells are currently mainly used for spacecraft applications but with the development of the Epitaxial Lift-Off process, the high purity semiconductor wafers that are required to produce these cells can be reused after separation of a single or even multiple cell structures [18-20]. This allows for a significant cost reduction, 


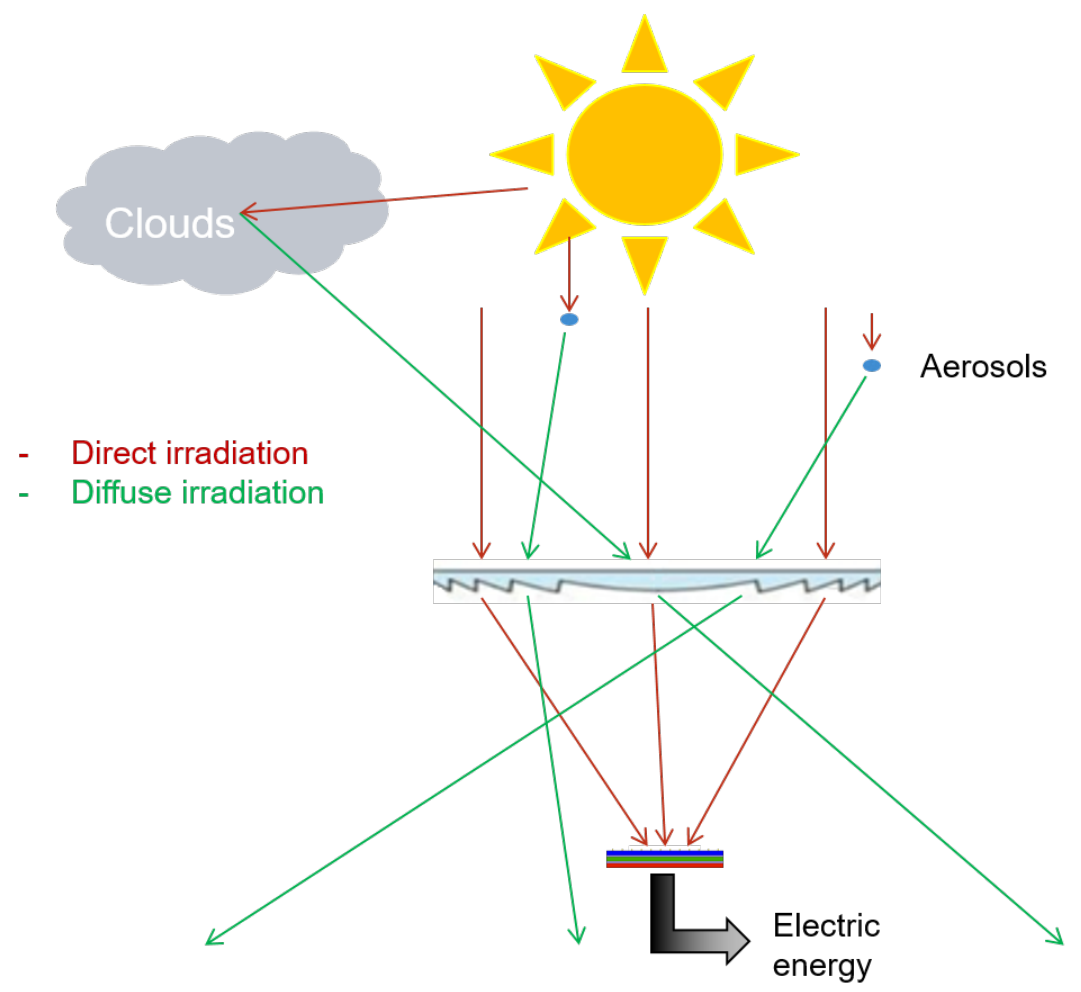

Fig. 1: A representation of a simple concentrating system. Direct illumination (red) is concentrated to the PV cell and converted into electrical energy, while diffuse illumination (green) is not concentrated and passes by the system. Here we seek to use this characteristic to employ the CPV as a façade incorporated daylight regulation system; removing the harsh direct component of the illumination while allowing the diffuse light inside the building.

increasing the utilization potential of these cells in other application areas such as CPV systems or high-end consumer products.

In ground-based CPV, systems are usually spaced far apart to avoid overlap [21,22]. However, when daylight regulation functionality is wanted, all harsh sunlight should be filtered at any time of the day. Thus individual CPV receivers must be in close proximity to each other. In this case adjoining panels will partially slide behind one another during tracking as further detailed in figure 4, so each panel casts a shadow on the one behind it. Therefore in this work, the electrical power generation of the three above mentioned CPV systems is studied in detail using outdoor measurements, while partial shading of the systems is applied. Furthermore, a parallel and series interconnection scheme of a multi-receiver system may have implications for the system tracking [15]. For that reason we propagate the shading along the $\mathrm{x}$ and $\mathrm{y}$ axis across the surface of the systems, to deconvolute any non identical effects on the systems electrical performance this entails. 

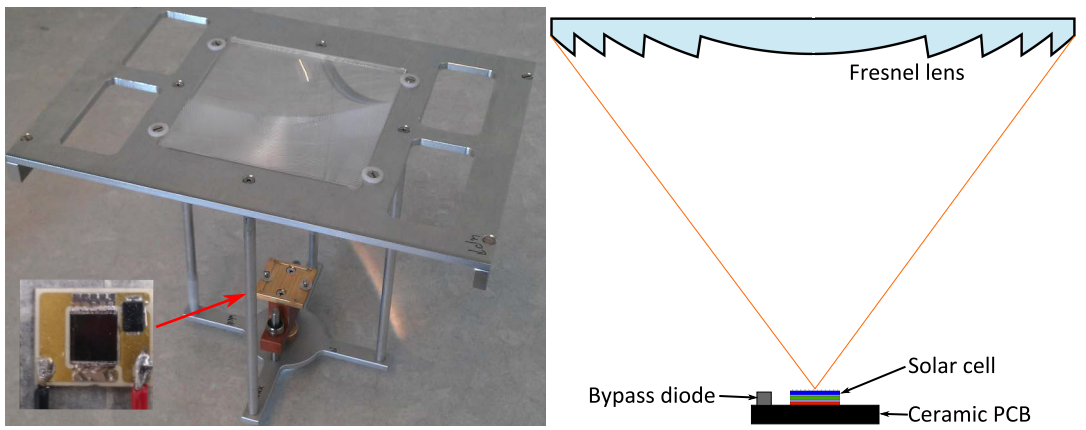

Fig. 2: The tested Fresnel lens based system. Left shows a photograph of the system whereupon the lens and receiver are mounted for outdoor tracked measurement. Right the structure of the receiver integrated with the lens.
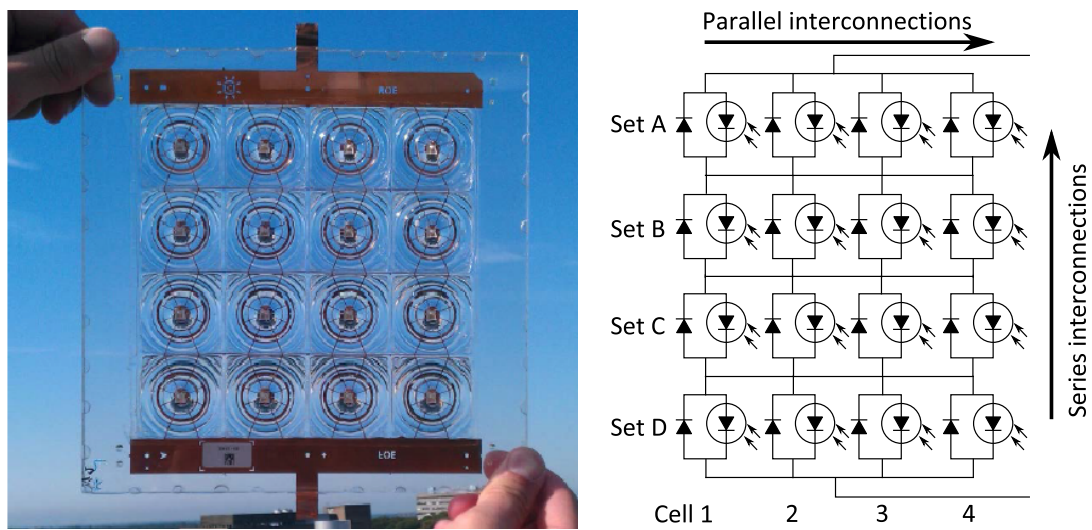

Fig. 3: The tested multi-receiver panel. Left a photograph of the panel. Right a representation of the electrical interconnection of the system where each receiver is represented by a photodiode in parallel with a bypass diode. Sets A - D consist of 4 parallel connected receivers each. The sets are in turn interconnected in series.

\section{Experimental}

\subsection{Device description}

The first device under test, shown in figure 2 features a $10 \mathrm{~cm} \mathrm{x} 10 \mathrm{~cm}$ Fresnel lens made of PMMA as a concentrating optic, which has a focal depth of $10 \mathrm{~cm}$. A $10 \mathrm{~mm} \times 10 \mathrm{~mm}$ TJ cell mounted on a ceramic printed circuit board (PCB) and equipped with a bypass diode is placed in the lens' focal point, giving the system a geometric concentration factor of 100 . Secondly a planar optic receiver is investigated, which consists of a $4 \mathrm{~cm} \mathrm{x} 4 \mathrm{~cm}$ planar focusing optic, a $1.3 \mathrm{~mm} \times 1.3 \mathrm{~mm}$ TJ III-V cell, a bypass diode, a copper heat sink and integrated wiring [15]. The benefit of using this type of receiver compared to more regular optics is that the optical system does not have an air cavity to account for the focal distance of the optics and thus is flat. This significantly enlarges its potential for utilization in building-integrated systems. The receiver has been described elsewhere [15] and will not be further detailed here. The receiver has a high geometrical concentration factor of 920 .

Finally a 16 receiver panel of these planar optics is investigated, which is shown in figure 3. It consists of a $4 \times 4$ array of planar optic receivers with integrated solar cell assembly, which are interconnected in 4 parallel strings that are in turn connected in series. In application, 
multiple panels will be enclosed in a transparent glass enclosure. Figure 4 shows a schematic representation of such a setup consisting of nine panels with $w$ and $h$ the width and height of a panel respectively, and $d_{h o r}$ and $d_{v e r t}$ the horizontal and vertical heart-to-heart distance between panels ${ }^{1}$. The system layout is optimized primarily with daylight regulation in mind,
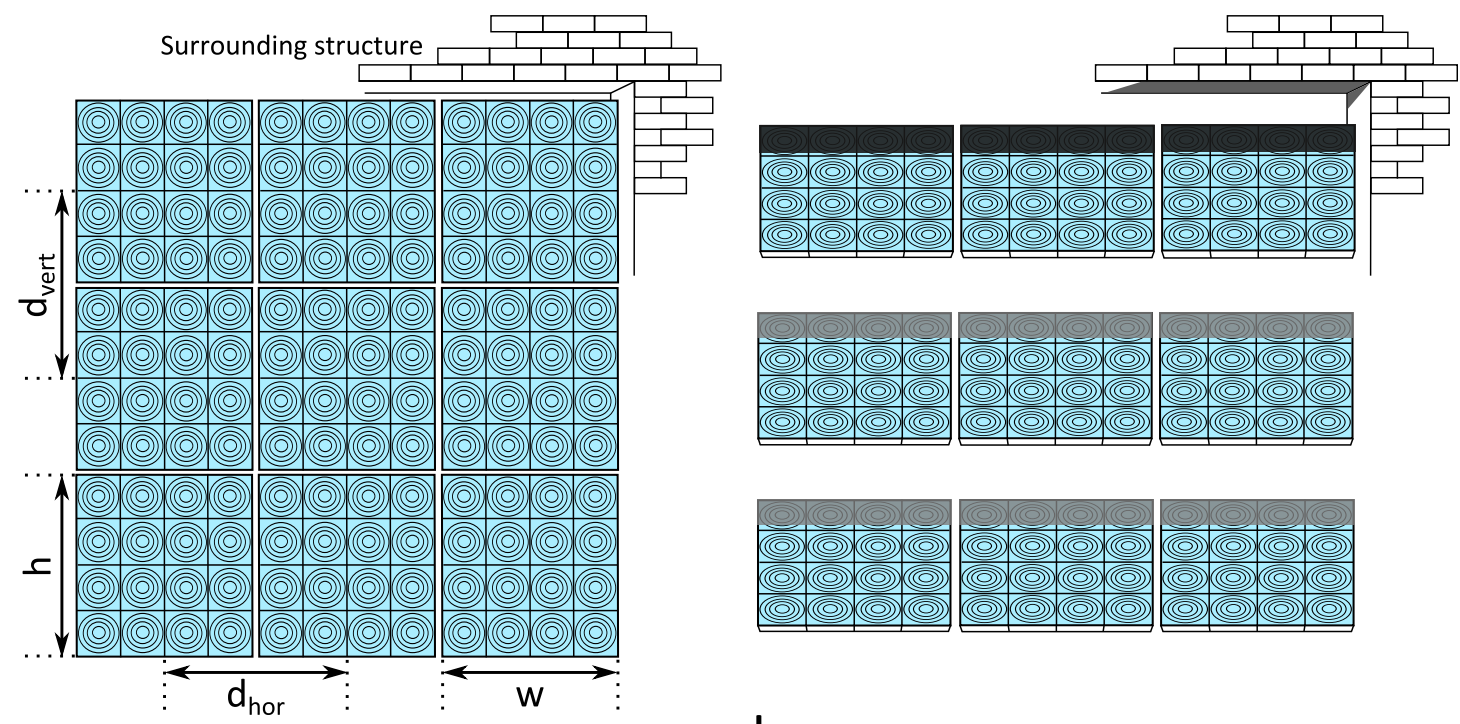

a
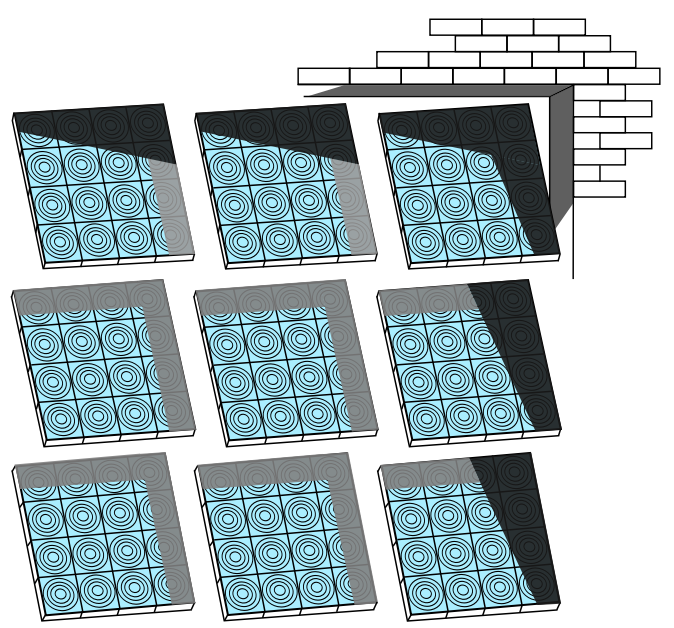

C

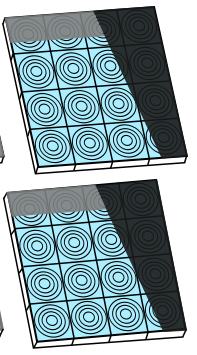

b
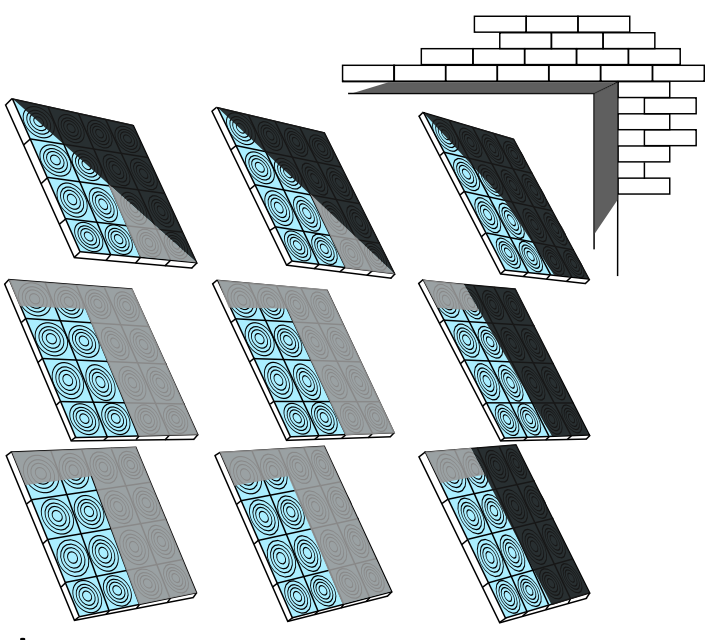

d

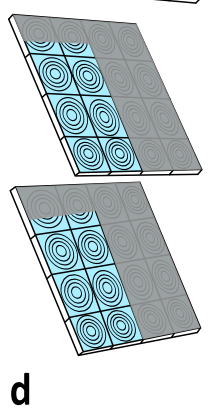

Fig. 4: Schematic representation of a nine panel system in a building façade oriented due south. The shadow cast on the panels by the building (black) and other panels (gray) is shown for different tracking positions. The sun position relative to the building is a) perpendicular, b) elevation $30^{\circ}$, azimuth $0^{\circ}$, c) elevation $30^{\circ}$, azimuth $-30^{\circ}$, and d) elevation $30^{\circ}$, azimuth $-60^{\circ}$. Note that azimuth is defined with respect to the south, and elevation of $90^{\circ}$ is defined as directly overhead.

in such a way that apparent full coverage of the façade is achieved at any time during the day as a function of the sun position. Thus, if sunlight is incident perpendicular to the façade, the

\footnotetext{
${ }^{1}$ From the figure can also be noted that in application, the system needs a space in front of the window to enable mechanical tracking.
} 
individual panels are located side by side, fully covering the façade without covering each other (thus $d_{\text {hor }}=w$ and $d_{\text {vert }}=h$ ). The system tracks the sun by tilting and rotating the individual panels. As can be seen from figure $4 \mathrm{~b}$, c, and d, during tracking a panel may be shadowed by the building (black) or another panel (gray). The shadowing by the building around the semitransparent system is highly dependent on the way it is incorporated in the façade and becomes less important as the system becomes increasingly larger. However, shading by adjacent panels is an intrinsic phenomenon in the above described concept. Therefore its influence on the electrical output of the system will be evaluated in detail in this study.

\subsection{Measuring procedure}

In order to determine the electrical energy production of the three systems, outdoor currentvoltage (I-V) measurements have been performed to closely approximate actual operating conditions. Aside from investigating the electrical performance of the systems, also shadowing experiments have been performed. A procedure similar to that of Rodrigo et.al. [23] has been used for the shadowing experiments. Although they used high concentration Fresnel based systems with secondary optics as opposed to our low concentration Fresnel lens system and high concentration flat plate systems, the primary optic shadowing procedure is applicable in all cases. Opaque metal plates were slid incrementally over the systems, causing a rectangular shadow shape. Of course, as can be seen from figure 4 in practice not only rectangular shadow shapes will be caused in the panels. Depending on the tracking position also diagonal shadows will be present. However, due to symmetry in the studied systems, application of a horizontal resp. vertical shadow on the optics allows for deconvolution of different sources of current generation loss. Data was collected on the performance of the entire panel, as well as the individual receivers. The latter was achieved by blocking all other receivers of the panel with an opaque metal plate. This approach is possible because of the integrated bypass diode in each receiver, which allows current to bypass a non-functioning cell. This was done on the single-receiver level in order to make a pure comparison between the work on a Fresnel lens based concentrator and the planar optic receiver considered here, as well as on the panel level to investigate the total device performance.

Outdoor urban I-V characterization of the panel was performed at the Applied Materials Science group of the Radboud University (Nijmegen, The Netherlands $51.82^{\circ} \mathrm{N} ; 5.87^{\circ} \mathrm{E}$ ). The setup is shown in figure 5. The panel was enclosed in a metal harness so it could be mounted on an EKO STR 22 Sun Tracker. The harness features grooved slots for the feeding of metal plates for controlled in situ shadowing of the panel. Ambient temperature and humidity are obtained by Campbell Scientific CR1000 measurement and control datalogger. Wind speed is measured by RM Young RM05103 wind meter. Global, horizontal and tracked insolation are monitored by EKO 402 pyranometers. Finally direct normal illumination (DNI) is measured with a Hukseflux DR02 pyrheliometer. In-field I-V characteristics are acquired using a Keithley 2601 source metre. I-V curves contain data on the parameters used to determine the solar cell quality and performance. Here, the considered parameters are the generated current in short-circuit conditions $\left(I_{S C}\right)$, cell potential in open circuit conditions $\left(V_{O C}\right)$, and the current and potential corresponding to maximum power output under load ( $I_{M P}$ and $V_{M P}$ respectively). From this and the DNI the maximum generated power:

$$
P_{M P}=I_{M P} \cdot V_{M P}
$$

and cell efficiency:

$$
\eta=\frac{P_{M P}}{D N I}
$$

are calculated. Experimental I-V data retrieval and processing are performed with ReRa Solutions Tracer 3 software. As multi-junction concentrator solar cells are influenced by changes in 


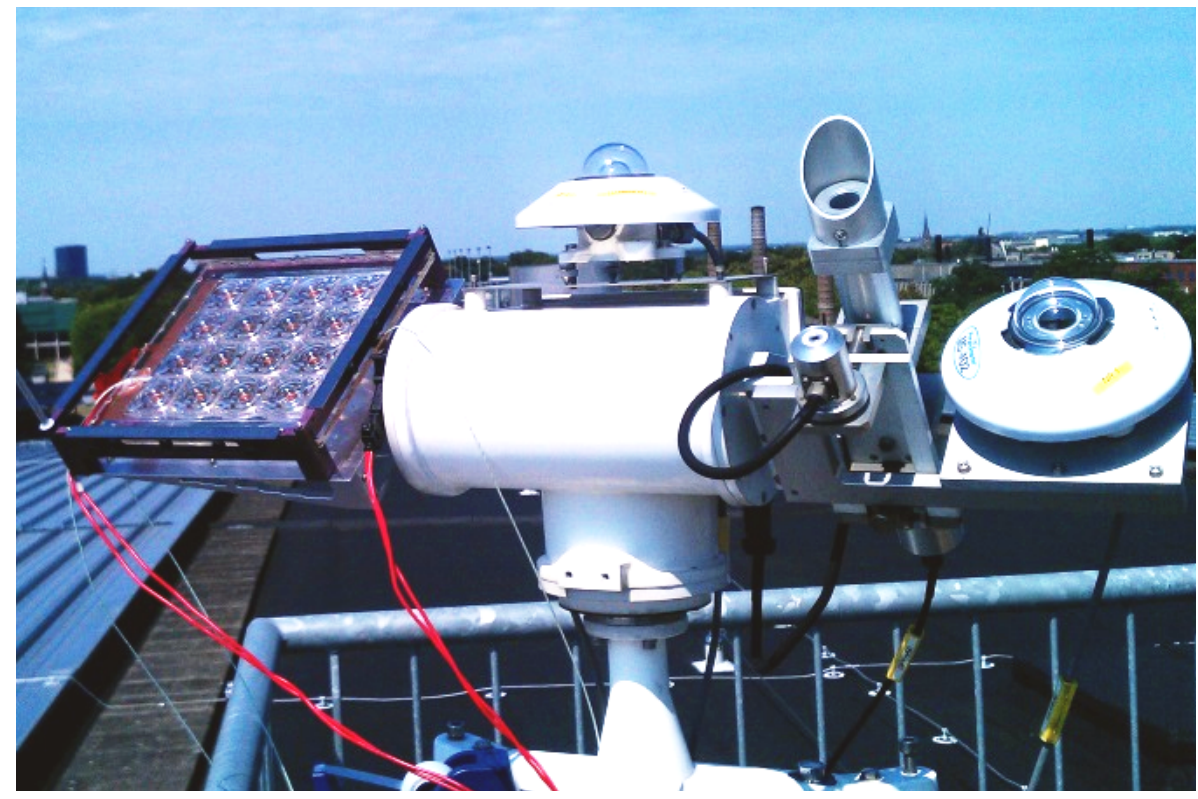

Fig. 5: The outdoor measurement setup with the multi-receiver flat plate system attatched to the tracker. Also shown are the pyrheliometer and pyranometers.

irradiance, cell temperature and incident light spectrum [24-26], measurements are performed in such a way as to keep these factors constant. For this purpose, measurements were performed on very clear days around noon so air mass, clouds, turbidity and precipitable water vapour can be considered stable during the measurements. Therefore the spectral distribution was considered constant across each measurement set taken in a limited time frame of maximally 30 minutes. Even so, cell temperature will strongly decrease with shading because the irradiance across the solar cell is reduced. Therefore, between I-V measurements the opaque plate is removed so the cell is fully illuminated. In order to keep cell temperature constant across the measurement series, curves are measured rapidly after the plate is replaced.

\section{Results}

\subsection{Fresnel lens system}

Figure 6 shows the electrical performance of the Fresnel lens based system for various shadow fractions s. The I-V curve for an unshaded lens (top blue curve in figure 6a) shows an $I_{S C}$ of $819 \mathrm{~mA}$ at the specified atmospheric conditions. For 1-sun illumination $\left(1000 \mathrm{~W} / \mathrm{m}^{2}\right)$ this would amount to a short-circuit current $I_{S C, F r e s n e l}^{\text {system }}$ of $830 \mathrm{~mA}$. From this and the cell's short circuit current measured in the lab: $I_{S C, F r e s n e l}^{c e l l}=13.4 \mathrm{~mA}$, the concentration factor of the Fresnel lens system is determined via:

$$
C=\frac{I_{\text {SC,Fresnel }}^{\text {system }}}{I_{S C, \text { Fresnel }}^{\text {cell }}}
$$

which yields $\mathrm{C}=62$ for this system. Given the geometric concentration factor of 100 for this system, the concentration efficiency of the system can be defined as:

$$
N=\frac{C}{C_{g e o}}
$$



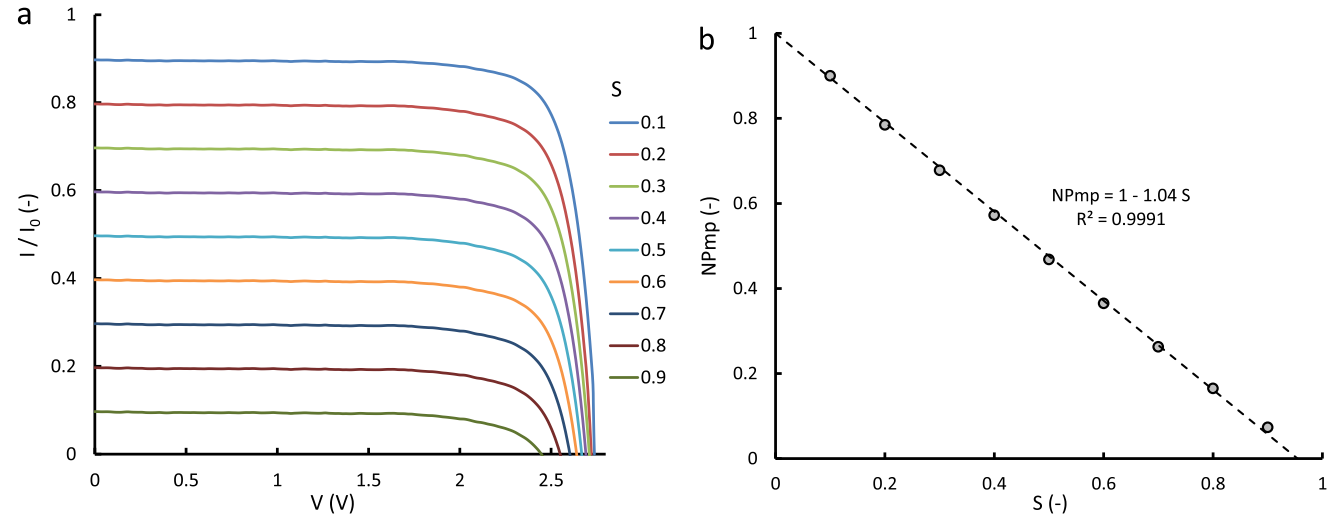

Fig. 6: Electrical power output of the Fresnel lens system for measuring conditions: DNI = $(0.987 \pm 0.007) \mathrm{kW} / \mathrm{m}^{2}, \mathrm{~T}=(15.2 \pm 0.3)^{\circ} \mathrm{C}, v_{\text {wind }}=(1.6 \pm 1.0) \mathrm{m} / \mathrm{s}$. a) $\mathrm{I}-\mathrm{V}$ curves for different shading factors show that $I_{S C}$ drops linearly as a function of shading factor while $V_{O C}$ shifts slightly, in accordance with the relative illumination. b) Normalized maximum power as a function of shading factor. The dotted line represents the best linear fit through the data points, starting at $N P_{M P}=1$
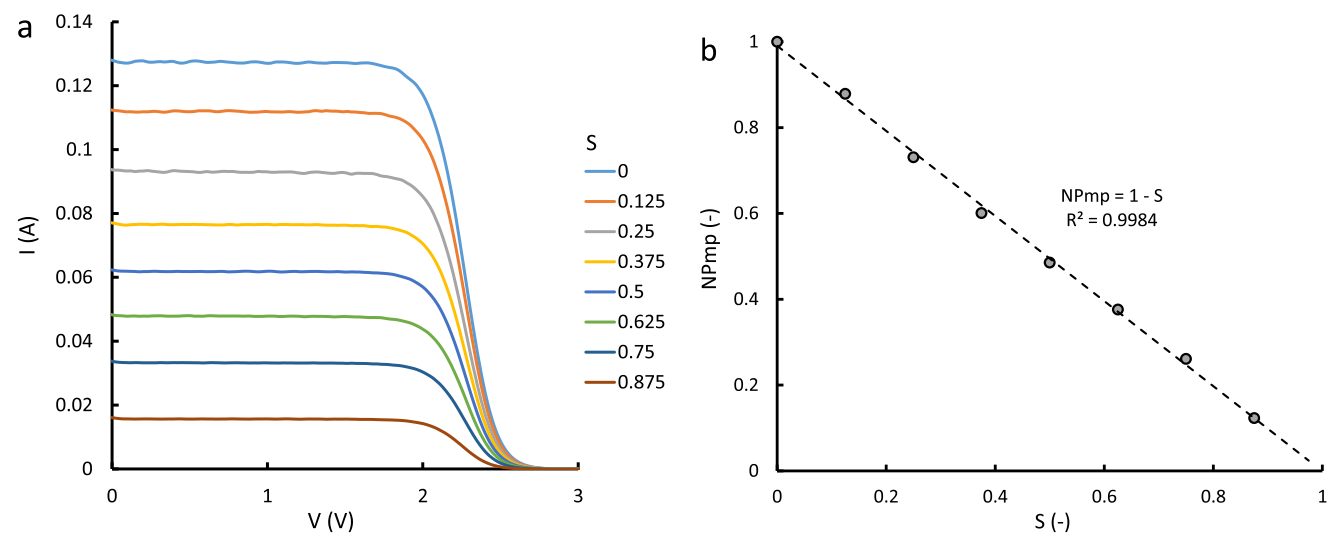

Fig. 7: Electrical output of receiver A1 (see figure 3) for measuring conditions: DNI $=(0.817 \pm$ $0.002) \mathrm{kW} / \mathrm{m}^{2}, \mathrm{~T}=(28.4 \pm 0.01)^{\circ} \mathrm{C}, v_{\text {wind }}=(2.2 \pm 1.0) \mathrm{m} / \mathrm{s}$. a) I-V curves for different shading factors. In the high voltage range, the curves show a tail; this is an effect of the incorporated bypass diodes. b) Normalized maximum power as a function of shading factor. The dotted line represents the best linear fit through the data points, starting at $N P_{M P}=1$.

which is 0.62 for this system.

The lens has been shaded for $\mathrm{s}=0,0.1, \ldots, 0.8,0.9$ and $\mathrm{I}-\mathrm{V}$ curves for each increment are shown in figure $6 \mathrm{a}$. For increasing shading factor, the effective illumination of the lens and thus the cell becomes less, and the data show the accompanying drop in generated current by the solar cell to be linear and proportional, in a very similar fashion as is described in [23] for a Fresnel lens system featuring a secondary optic element (SOE). Maximum power values, normalized to the unshadowed case $N P_{M P}$, are shown in figure $6 \mathrm{~b}$. Here we also find a linear decrease in maximum power for increasing shadow factor, similar to what is described for a higher concentration Fresnel system with SOE. From the fit, a loss of performance in the order of $4 \%$ is noted here, while for a system that includes a SOE and a heat sink this amounts to 
$0 \%$. This difference can be attributed to the homogenizing properties of the SOE, as the other measuring conditions are similar for both systems.

\subsection{Single planar optic concentrator}

The performance of individual planar optic receivers has been studied without disassembling the panel, by covering 15 of the 16 receivers of the panel with an opaque plate, to determine variations in performance needed for understanding the total I-V curves of the entire system as will be described in section 3.3. The I-V curve for an unshaded receiver (top blue curve in figure $7 \mathrm{a}$ ) shows an $I_{S C}$ of $129 \mathrm{~mA}$ at the specified atmospheric conditions. For 1-sun illumination $\left(1000 \mathrm{~W} / \mathrm{m}^{2}\right)$ this would amount to a short-circuit current $I_{S C, p l a n a r}^{\text {system }}$ of $156 \mathrm{~mA}$. From this and cell specifications for the short circuit current $I_{S C \text {,planar }}^{\text {cell }}=0.24 \mathrm{~mA}$, the concentration factor of the planar optic receiver is determined via equation (3) - where the "Fresnel" subscripts are exchanged for "planar" - to be 647 for this particular receiver. When similar calculations are performed for all receivers, $\mathrm{C}$ values in the range of 610 to 710 are found. Via equation (4) this amounts to a concentration efficiency for the planar optic system of $\mathrm{N}=[0.66-0.77]$. Therefore the functionality of this flat plate concentrator may be considered at least equal to a common Fresnel lens based system.

Additionally, to investigate the sensitivity of the planar optic concentrator to inhomogeneous illumination, the unblocked receiver has been incrementally shaded by a metal plate. Shading factors of $\mathrm{s}=0,0.125,0.250, \ldots, 0.750,0.875$ were investigated. Typical I-V data for one receiver are shown in figure 7a. For increasing shading factor, the effective illumination of the receiver becomes less, and the data show the accompanying drop in generated current by the solar cell to be linear and proportional, in a very similar fashion as both the low concentration Fresnel lens bases system discussed above, and high concentration Fresnel systems described in [23]. Maximum power values, normalized to the unshadowed case $N P_{M P}$, are shown in figure $7 \mathrm{~b}$. Here we also find a linear decrease in maximum power for increasing shadow factor. Therefore we conclude that the planar concentrating optic handles inhomogeneous or partial illumination very well, and therefore it is suitable for use in the same conditions in which a Fresnel optic of similar concentration factor can be used.

\subsection{Flat plate panel}

Figure 8 shows the I-V curves of the entire 16 receiver panel, as shown in figure 3 , during outdoor measurement. The curves feature four distinct steps in the current for increasing voltage. These arise because of the parallel interconnection of the receivers, in combination with a slight current mismatch between the individual solar cells. As the panel consists of four sets of four cells connected in parallel, each set generates a total current equal to the sum of its consistuent cell currents, which for the panel under investigation varies slightly between the sets. The total generated current for any given voltage is then governed by the series interconnection between sets. Therefore at low voltage we measure the current of the best performing set, while at higher voltage the current is limited by weaker performing sets, resulting in the stepped shape of the total I-V characteristic with steps appearing at the $V_{O C}$ of a set - or the sum of $V_{O C}$ 's from multiple sets. During operation the system is kept at its maximum power point $\left(P_{M P}\right)$ so electricity generation is maximum at all times. For an unshaded system $P_{M P}$ occurs at $10.54 \mathrm{~V}$. As can be seen from figure $8 \mathrm{a}$, this means that the system operates at a current limited by the set that shows the least performance, as can be expected from the series connections of the sets. During testing the panel showed an average maximum power output of $4.4 \pm 0.1 \mathrm{~W}$ and a conversion efficiency of $24 \pm 0.6 \%$.

The interconnection between several components in the BICPV panel also has consequences for the electrical output if shading of the system is present. Due to the presence of both parallel and series interconnections, various shapes of shadows will have different effects on the performance 

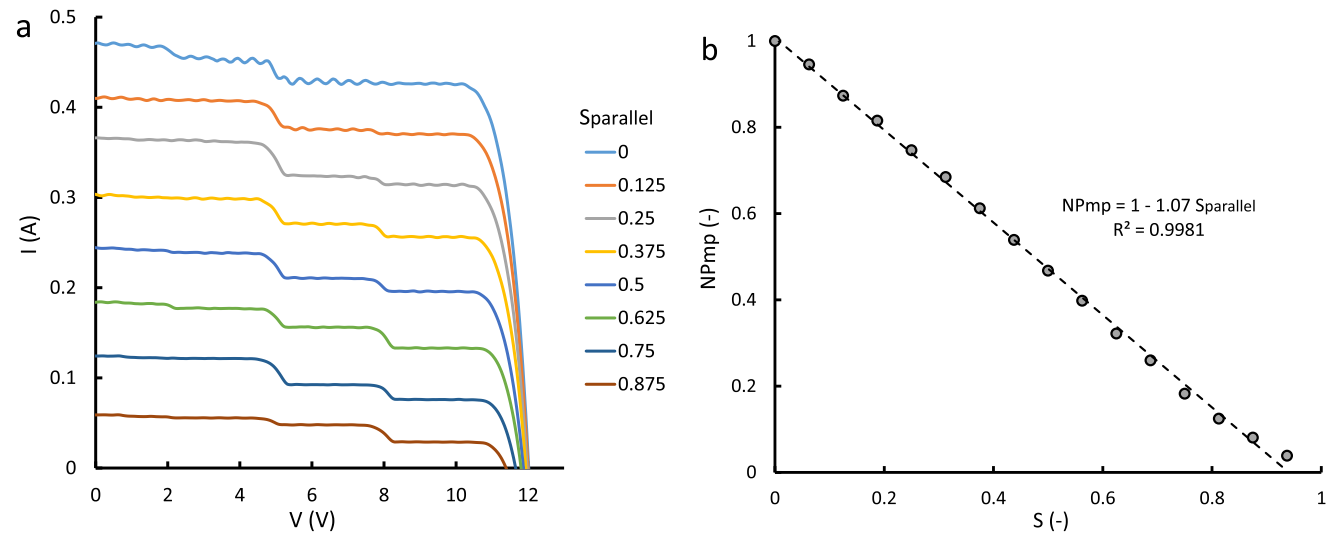

Fig. 8: Electrical output of the panel for different shading factors along the line of the parallel interconnections. Measuring conditions: DNI $=(0.794 \pm 0.003) \mathrm{kW} / \mathrm{m}^{2}, \mathrm{~T}=(27.0 \pm$ $0.4)^{\circ} \mathrm{C}, v_{\text {wind }}=(2.7 \pm 1.0) \mathrm{m} / \mathrm{s}$. a) I-V curves for different shading factors show that the $I_{S C}$ drops quite linearly as a function of shading factor while $V_{O C}$ remains roughly constant, as can be expected for diminishing illumination. b) Normalized maximum power as a function of system shading. The dotted line represents the best linear fit through the data points, starting at $N P_{M P}=1$.
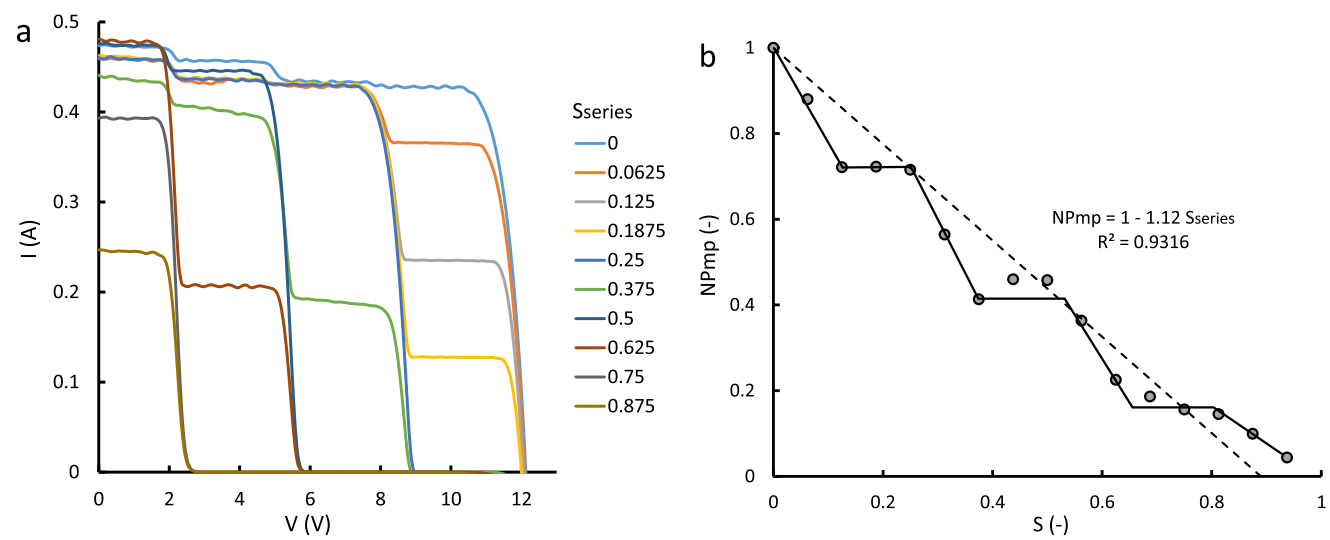

Fig. 9: Electrical output of the panel for different shading factors along the line of the series interconnections. Measuring conditions: $\mathrm{DNI}=(0.809 \pm 0.002) \mathrm{kw} / \mathrm{m}^{2}, \mathrm{~T}=(27.2 \pm$ $0.3)^{\circ} \mathrm{C}, v_{\text {wind }}=(2.4 \pm 0.8) \mathrm{m} / \mathrm{s}$. a) I-V curves for different shading factors show that the $I_{S C}$ drops quite linearly while $V_{O C}$ remains roughly constant, unless a parallel string is completely blocked, at which point the $V_{O C}$ drops proportionally, causing a quite erratic characteristic. b) Normalized maximum power as a function of system shading showing a stepwise decrease as is emphasized by the continuous line through the data points. The dotted line represents the best linear fit through the data points, starting at $N P_{M P}=1$.

of the panel. To investigate this effect, the panel was incrementally shadowed in two directions; along the parallel connections, and along the series connections. Sixteen increments in shading have been applied, so that each set of adjoining receivers is shaded $\mathrm{s}=0,0.25,0.50,0.75,1$. The I-V data for shading the panel along the parallel direction are shown in figure 8a, where each successive downward step represents an increase in shading. The data show a linear decay in generated current as a function of increased shading, which is expected as each set is shaded to an equal amount. Figure $8 \mathrm{~b}$ shows that the generated maximum power also decays in a linear 
fashion across almost the entire series, indicating that $V_{M P}$ remains roughly constant, unless the panel is nearly entirely shaded, allowing straightforward maximum power point (MPP) tracking in the application.

The I-V curves for shading along the series direction are shown in figure 9a. Here, a more severe effect is observed. In this case one set is shaded fully before the next receives any shading, so the current level of individual steps in the I-V curve decreases consecutively as sets are excluded from contributing to the current generation. This not only leads to a decrease in $P_{M P}$, but also in $V_{M P}$ for increasing shading factors as shown in figure $9 \mathrm{~b}$. Furthermore, the maximum power output decreases in a stepwise fashion and more strongly than would be expected from a linear dependency. A linear fit through the data points indicates that on average, the difference between the observed power output and a linear decrease is $12 \%$ of the maximum output of the system. Also, distinct steps in $V_{M P}$ can be noted, which occur when one set is entirely deprived of illumination and thus no longer provides voltage. This shifting of $V_{M P}$ is clearly of consequence for the MPP tracking of the panel. From this can be concluded that for this panel a shadow of arbitrary shape will at best reduce the electrical output proportionally to the shadowed area and at worst cause an additional $12 \%$ loss of performance. More generally it can be noted that any BICPV system is less robust to external shading if more series interconnections are present. If there are no restrictions on the system voltage, reduction of the amount of series interconnections is suggested to be beneficial for the overall system performance in areas where system shading is common.

\section{Conclusions}

The concept of using semi-transparent CPV elements for a façade incorporated daylight regulation system, as well as an energy source has been introduced. Because in this context, adjacent CPV receivers will partially slide behind and shadow one another, the electrical power output of three model CPV systems when partially shadowed has been investigated. Outdoor I-V measurements were used to approximate the actual working conditions of a BICPV system closely, and to analyze the current and power generation parameters of the systems in detail. A novel concentrating panel based on flat planar optics that offers the benefit of lacking an air cavity to account for optical focal depth is compared to a reference Fresnel lens based system, and the promise of these flat concentrators for use in BICPV applications has been shown.

It has been shown that the electrical power output of a Fresnel lens based concentrator system without secondary optic has a linear dependency on shadow factor. A loss of performance in the order of $4 \%$ has been observed in this system, compared to $0 \%$ previously reported for a system featuring a SOE. The concentration efficiency of this system is determined to be 0.62.

The novel planar optic concentrator system with integrated TJ solar cell has also been regarded. The current and power generation parameters of these flat receivers are similar to those of a Fresnel lens based system of comparable concentration. High concentration factors exceeding 610 have been experimentally determined for these receivers, much larger values than other systems in the BICPV field where concentrations less than 50 are more common. A concentration efficiency in the range of $0.66-0.77$ is noted for the planar optic concentrator, which is in the same range as the Fresnel lens system. The net conversion efficiency of this system (in standard test conditions) was determined to be in the order of $24 \%$. Shadowing of the optic of a single receiver has been shown to lead to a proportionate loss in generated power, showing an extra loss of performance of $0 \%$, like Fresnel lens based systems using a SOE. The electrical power generation is comparable to a lens based system, but these concentrators are flat, and allow easier daylight regulation than more bulky lens based CPV systems. Therefore these planar optic concentrators can be considered very adequate for use in BICPV.

For a multi-receiver panel with interconnections both in series and in parallel, the maximum 
output power shows a disproportionally large decrease as a function of shadow fraction: between $7 \%$ along the parallel interconnections, and up to $12 \%$ in the series direction. This decrease can be fully attributed to the interconnection design of the panel as the single optics have been shown not to suffer from such performance loss. Therefore this can be mitigated or alleviated by making alterations to system or building design for specific applications. This can for instance be achieved by reducing the amount of series connections, or orienting the panels such that shadowing along the series direction is minimal.

\section{Intellectual property}

Note that this publication illustrates principles and technologies which are part of the intellectual property of Morgan Solar. The technology discussed in this paper falls under various international patents and patent applications as specified in [15].

\section{Acknowledgements}

The authors acknowledge STW for financial support for this project from Valorization Grant number 13226.

\section{References}

[1] European Parliament. Directive 2010/31/EU of the european parliament and of the council of 19 may 2010 on the energy performance of buildings, 2010.

[2] João Gomes, Linkesh Diwan, Ricardo Bernardo, and Björn Karlsson. Minimizing the impact of shading at oblique solar angles in a fully enclosed asymmetric concentrating pvt collector. Energy Procedia, 57:2176-2185, 2014.

[3] Noboru Yamada, Kosuke Kanno, Kentaro Hayashi, and Toru Tokimitsu. Performance of see-through prism CPV module for window integrated photovoltaics. Optics express, 19(104):A649-A656, 2011.

[4] Daniel Chemisana. Building integrated concentrating photovoltaics: a review. Renewable and Sustainable Energy Reviews, 15(1):603-611, 2011.

[5] Ruei-Tang Chen, Joseph Lik Hang Chau, and Gan-Lin Hwang. Design and fabrication of diffusive solar cell window. Renewable Energy, 40(1):24-28, 2012.

[6] N Aste, LC Tagliabue, C Del Pero, D Testa, and R Fusco. Performance analysis of a large-area luminescent solar concentrator module. Renewable Energy, 76:330-337, 2015.

[7] Nabin Sarmah and Tapas K Mallick. Design, fabrication and outdoor performance analysis of a low concentrating photovoltaic system. Solar Energy, 112:361-372, 2015.

[8] Aggelos Zacharopoulos, Philip Charles Eames, Dominic McLarnon, and Brian Norton. Linear dielectric non-imaging concentrating covers for PV integrated building facades. Solar Energy, 68(5):439-452, 2000.

[9] Hasan Baig. Enhancing performance of building integrated concentrating photovoltaic systems. 2015.

[10] Hasan Baig, Nazmi Sellami, Daniel Chemisana, Joan Rosell, and Tapas K Mallick. Performance analysis of a dielectric based 3d building integrated concentrating photovoltaic system. Solar Energy, 103:525-540, 2014. 
[11] Hasan Baig, Nazmi Sellami, and Tapas K Mallick. Performance modeling and testing of a building integrated concentrating photovoltaic (BICPV) system. Solar Energy Materials and Solar Cells, 134:29-44, 2015.

[12] Daniel Chemisana, Ma Victoria Collados, Manuel Quintanilla, and Jesús Atencia. Holographic lenses for building integrated concentrating photovoltaics. Applied Energy, 110:227$235,2013$.

[13] D Chemisana, JI Rosell, A Riverola, and Chr Lamnatou. Experimental performance of a fresnel-transmission PVT concentrator for building-façade integration. Renewable Energy, 85:564-572, 2016.

[14] Philippe Voarino, César Domínguez, Roy Bijl, and Peter Penning. Optical performance analysis of a novel tracking-integrated concentrator through ray tracing. In 10th International Conference on Concentrator Photovoltaic Systems, AIP Conf. Proc. 1616, pages 215-219, 2014.

[15] J.P. Morgan, S. Myrskog, B. Barnes, M. Sinclair, and N. Morris. Sunlight concentrating and harvesting device, 2014 - 2015. US Patent App. 14/196,618; 14/215,913; 14/218,025; 14/218,649; 14/217,998; 14/196,291; US 14/196,523. WO Patent App. PCT/US14/31002; PCT/CA2014/000167; PCT/CA2014/050168; PCT/CA2014/000263.

[16] Vjačeslav M Andreev and Antonio Luque. Concentrator photovoltaics. Springer, 2007.

[17] Ignacio Luque-Heredia, Goulven Quéméré, Rafael Cervantes, Olivier Laurent, Emmanuele Chiappori, and Jing Ying Chong. The sun tracker in concentrator photovoltaics. In Next Generation of Photovoltaics, pages 61-93. Springer, 2012.

[18] GJ Bauhuis, P Mulder, EJ Haverkamp, JJ Schermer, E Bongers, G Oomen, W Köstler, and G Strobl. Wafer reuse for repeated growth of iii-v solar cells. Progress in Photovoltaics: Research and Applications, 18(3):155-159, 2010.

[19] MMAJ Voncken, JJ Schermer, GJ Bauhuis, P Mulder, and PK Larsen. Multiple release layer study of the intrinsic lateral etch rate of the epitaxial lift-off process. Applied Physics A, 79(7):1801-1807, 2004.

[20] Jongseung Yoon, Sungjin Jo, Ik Su Chun, Inhwa Jung, Hoon-Sik Kim, Matthew Meitl, Etienne Menard, Xiuling Li, James J Coleman, Ungyu Paik, et al. Gaas photovoltaics and optoelectronics using releasable multilayer epitaxial assemblies. Nature, 465(7296):329-333, 2010.

[21] P Rodrigo, Eduardo F Fernández, F Almonacid, and PJ Pérez-Higueras. A simple accurate model for the calculation of shading power losses in photovoltaic generators. Solar Energy, 93:322-333, 2013.

[22] P Rodrigo, S Gutiérrez, Ramiro Velázquez, Eduardo F Fernández, F Almonacid, and PJ Pérez-Higueras. A methodology for the electrical characterization of shaded high concentrator photovoltaic modules. Energy, 89:768-777, 2015.

[23] P Rodrigo, Eduardo F Fernández, F Almonacid, and PJ Pérez-Higueras. Outdoor measurement of high concentration photovoltaic receivers operating with partial shading on the primary optics. Energy, 61:583-588, 2013.

[24] G Siefer, P Abbott, C Baur, T Schlegl, and AW Bett. Determination of the temperature coefficients of various iii-v solar cells. In Proceedings of the 20th European Photovoltaic Solar Energy Conference, Barcelona, Spain, pages 495-498, 2005. 
[25] Eduardo F Fernández, G Siefer, M Schachtner, AJ García Loureiro, and P Pérez-Higueras. Temperature coefficients of monolithic iii-v triple-junction solar cells under different spectra and irradiance levels. In American Institute of Physics Conference Series, volume 1477, pages 189-193, 2012.

[26] Gerald Siefer and Andreas W Bett. Analysis of temperature coefficients for iii-v multi-junction concentrator cells. Progress in Photovoltaics: Research and Applications, 22(5):515-524, 2014. 